\title{
LINEARIZATION OF BOUNDED HOLOMORPHIC MAPPINGS ON BANACH SPACES
}

\author{
JORGE MUJICA
}

\begin{abstract}
The main result in this paper is the following linearization theorem. For each open set $U$ in a complex Banach space $E$, there is a complex Banach space $G^{\infty}(U)$ and a bounded holomorphic mapping $g_{U}: U \rightarrow G^{\infty}(U)$ with the following universal property: For each complex Banach space $F$ and each bounded holomorphic mapping $f: U \rightarrow F$, there is a unique continuous linear operator $T_{f}: G^{\infty}(U) \rightarrow F$ such that $T_{f} \circ g_{U}=f$. The correspondence $f \rightarrow T_{f}$ is an isometric isomorphism between the space $H^{\infty}(U ; F)$ of all bounded holomorphic mappings from $U$ into $F$, and the space $L\left(G^{\infty}(U) ; F\right)$ of all continuous linear operators from $G^{\infty}(U)$ into $F$. These properties characterize $G^{\infty}(U)$ uniquely up to an isometric isomorphism. The rest of the paper is devoted to the study of some aspects of the interplay between the spaces $H^{\infty}(U ; F)$ and $L\left(G^{\infty}(U) ; F\right)$.
\end{abstract}

This paper consists of five sections. In $\S 1$ we establish our notation and terminology. In $\S 2$ we prove the aforementioned linearization theorem. In $\S 3$ we translate certain properties of a mapping $f \in H^{\infty}(U ; F)$ into properties of the corresponding operator $T_{f} \in L\left(G^{\infty}(U) ; F\right)$. We show, for instance, that $f$ has a relatively compact range if and only if $T_{f}$ is a compact operator.

In $\S 4$ we give a seminorm characterization of the unique locally convex topology $\tau_{\gamma}$ on $H^{\infty}(U ; F)$ such that the correspondence $f \rightarrow T_{f}$ is a topological isomorphism between the spaces $\left(H^{\infty}(U ; F), \tau_{\gamma}\right)$ and $\left(L\left(G^{\infty}(U) ; F\right), \tau_{c}\right)$, where $\tau_{c}$ denotes the compact-open topology.

Finally, in $\S 5$ we use the preceding results to establish necessary and sufficient conditions for the spaces $G^{\infty}(U)$ and $H^{\infty}(U)$ to have the approximation property. These are holomorphic analogues of classical results of A. Grothendieck [8], and complement results of R. Aron and M. Schottenloher [2]. We show, in particular, that if $U$ is a balanced, bounded, open set in a complex Banach space $E$, then $G^{\infty}(U)$ has the approximation property if and only if $E$ has the approximation property. We also show that if $U$ is an arbitrary open set in a complex Banach space $E$, then $H^{\infty}(U)$ has the approximation property if and only if, for each complex Banach space $F$, each mapping in $H^{\infty}(U ; F)$ with a relatively compact range can be uniformly approximated on $U$ by mappings in $H^{\infty}(U ; F)$ with finite-dimensional range. Since it is still unknown whether

Received by the editors April 10, 1989. $46 \mathrm{E} 10$.

1980 Mathematics Subject Classification (1985 Revision). Primary 46G20, 46E15; Secondary 
the space $H^{\infty}(\Delta)$ has the approximation property, where $\Delta$ denotes the open unit disc, this result may be of some use in this connection.

I wish to thank Raymundo Alencar, Mário C. Matos and João B. Prolla for some helpful discussions while this paper was being prepared.

\section{NOTATION AND TERMINOLOGY}

Throughout this paper the symbols $\mathbb{R}$ and $\mathbb{C}$ represent the field of all real numbers and the field of all complex numbers, respectively. The set of all positive integers is denoted by $\mathbb{N}$, whereas the set $\mathbb{N} \cup\{0\}$ is denoted by $\mathbb{N}_{0}$.

The letters $E$ and $F$ always represent complex Banach spaces. The letter $U$ denotes a nonvoid open subset of $E$, whereas the symbol $U_{E}$ represents the open unit ball of $E$.

$L(E ; F)$ denotes the vector space of all continuous linear operators from $E$ into $F$. Unless stated otherwise, $L(E ; F)$ is endowed with its natural norm topology. We write $E^{\prime}$ instead of $L(E ; \mathbb{C})$ for the dual of $E$.

$P(E ; F)$ denotes the vector space of all continuous polynomials from $E$ into $F$. For each $m \in \mathbb{N}_{0}, P\left({ }^{m} E ; F\right)$ denotes the subspace of all $m$-homogeneous members of $P(E ; F)$. Unless stated otherwise, $P\left({ }^{m} E ; F\right)$ is endowed with its natural norm topology. When $F=\mathbb{C}$ we write $P(E)$ instead of $P(E ; \mathbb{C})$, and $P\left({ }^{m} E\right)$ instead of $P\left({ }^{m} E ; \mathbb{C}\right)$.

$H(U ; F)$ denotes the vector space of all holomorphic mappings from $U$ into $F$, and $H^{\infty}(U ; F)$ denotes the subspace of all bounded members of $H(U ; F)$. Unless stated otherwise, $H^{\infty}(U ; F)$ is endowed with the sup norm topology. When $F=\mathbb{C}$ we write $H(U)$ instead of $H(U ; \mathbb{C})$, and $H^{\infty}(U)$ instead of $H^{\infty}(U ; \mathbb{C})$.

For each $f \in H(U ; F), a \in U$ and $m \in \mathbb{N}_{0}, P^{m} f(a) \in P\left({ }^{m} E ; F\right)$ denotes the $m$ th term of the Taylor series of $f$ at $a$.

If $X$ is a locally convex space, then $X_{b}^{\prime}$ (resp. $X_{c}^{\prime}$ ) denotes the dual $X^{\prime}$ of $X$, endowed with the topology of uniform convergence on the bounded (resp. compact) subsets of $X$.

If $X$ is a topological space, then $C(X ; F)$ denotes the vector space of all continuous mappings from $X$ into $F$, and $\tau_{c}$ denotes the compact-open topology on $C(X ; F)$, or on any subset of $C(X ; F)$.

We refer to the books of S. Dineen [6] or the author [13] for the properties of polynomials and holomorphic mappings on infinite-dimensional spaces; to the book of J. Lindenstrauss and L. Tzafriri [10] for the theory of Banach spaces; and to the books of J. Horvath [9] or H. H. Schaefer [16] for the theory of topological vector spaces.

\section{LINEARIZATION OF BOUNDED HOLOMORPHIC MAPPINGS}

Let $U$ be an open subset of a Banach space $E$. As pointed out in S. Dineen's book [6, p. 417], a theorem of K. F. $\mathrm{Ng}$ [14] yields a Banach space $G^{\infty}(U)$ whose dual is isometrically isomorphic to $H^{\infty}(U)$. A refinement of this idea leads to the linearization theorem announced in the abstract. 
2.1. Theorem. Let $U$ be an open subset of a Banach space $E$. Then there are a Banach space $G^{\infty}(U)$ and a mapping $g_{U} \in H^{\infty}\left(U ; G^{\infty}(U)\right)$ with the following universal property: For each Banach space $F$ and each mapping $f \in$ $H^{\infty}(U ; F)$, there is a unique operator $T_{f} \in L\left(G^{\infty}(U) ; F\right)$ such that $T_{f} \circ g_{U}=$ $f$. The correspondence

$$
f \in H^{\infty}(U ; F) \rightarrow T_{f} \in L\left(G^{\infty}(U) ; F\right)
$$

is an isometric isomorphism. These properties characterize $G^{\infty}(U)$ uniquely up to an isometric isomorphism.

Proof. Let $B_{U}$ denote the closed unit ball of $H^{\infty}(U)$. By the Ascoli theorem $B_{U}$ is a compact subset of $H^{\infty}(U)$ for the compact-open topology $\tau_{c}$. Let $G^{\infty}(U)$ denote the closed subspace of all linear functionals $u \in H^{\infty}(U)^{\prime}$ such that $u \mid\left(B_{U}, \tau_{c}\right)$ is continuous. Let

$$
J_{U}: f \in H^{\infty}(U) \rightarrow \hat{f} \in G^{\infty}(U)^{\prime}
$$

denote the evaluation mapping, that is, $\hat{f}(u)=u(f)$ for every $f \in H^{\infty}(U)$ and $u \in G^{\infty}(U)$. By the $\mathrm{Ng}$ theorem [14], $J_{U}$ is an isometric isomorphism. Let

$$
g_{U}: x \in U \rightarrow \delta_{x} \in G^{\infty}(U)
$$

denote the evaluation mapping, that is, $\delta_{x}(f)=f(x)$ for every $x \in U$ and $f \in H^{\infty}(U)$. Since

$$
\left(J_{U} f\right) \circ g_{U}(x)=f(x),
$$

for every $x \in U$ and $f \in H^{\infty}(U)$, we see that $g_{U}$ is weakly holomorphic, and therefore holomorphic (see [13, p. 65, Theorem 8.12]). Since $\left\|\delta_{x}\right\|=1$ for every $x \in U$, we conclude that $g_{U} \in H^{\infty}\left(U ; G^{\infty}(U)\right)$.

Let ${ }^{0}$ indicate polars with respect to the dual system $\left\langle G^{\infty}(U), G^{\infty}(U)^{\prime}\right\rangle$. We claim that the closed unit ball $J_{U}\left(B_{U}\right)^{0}$ of $G^{\infty}(U)$ coincides with the closed, convex, balanced hull $\bar{\Gamma} g_{U}(U)$ of $g_{U}(U)$. In particular, $g_{U}(U)$ generates a dense subspace of $G^{\infty}(U)$. Indeed, since $J_{U}$ maps $H^{\infty}(U)$ onto $G^{\infty}(U)^{\prime}$, one can readily check that

$$
J_{U}\left(B_{U}\right)=g_{U}(U)^{0}
$$

and therefore

$$
J_{U}\left(B_{U}\right)^{0}=g_{U}(U)^{00}=\bar{\Gamma} g_{U}(U),
$$

by the bipolar theorem, as asserted.

We claim that the pair $\left(G^{\infty}(U), g_{U}\right)$ has the required universal property. If $f \in H^{\infty}(U)$, then we define $T_{f}=J_{U} f \in G^{\infty}(U)^{\prime}$ and the desired conclusion follows from (2.1). If $f \in H^{\infty}(U ; F)$, then we define $T_{f}: G^{\infty}(U) \rightarrow F^{\prime \prime}$ by

$$
\left(T_{f} u\right)(\psi)=u(\psi \circ f) \text {, }
$$

for every $u \in G^{\infty}(U)$ and $\psi \in F^{\prime}$. Clearly $T_{f} \in L\left(G^{\infty}(U) ; F^{\prime \prime}\right)$ and $\left\|T_{f}\right\|=$ $\|f\|$. Furthermore,

$$
\left(T_{f} \delta_{x}\right)(\psi)=\psi \circ f(x)
$$


for every $x \in U$ and $\psi \in F^{\prime}$, and therefore $T_{f} \delta_{x}=f(x) \in F$ for every $x \in U$. Since the evaluations $\delta_{x}$, with $x \in U$, generate a dense subspace of $G^{\infty}(U)$, it follows that $T_{f} \in L\left(G^{\infty}(U) ; F\right)$. The uniqueness of $T_{f}$ follows also from the fact that $g_{U}(U)$ generates a dense subspace of $G^{\infty}(U)$.

Finally, the uniqueness of $G^{\infty}(U)$ up to an isometric isomorphism follows from the universal property, together with the isometry $\left\|T_{f}\right\|=\|f\|$. This completes the proof.

2.2. Remark. In the proof of Theorem 2.1 we have shown that the closed unit ball of $G^{\infty}(U)$ coincides with $\bar{\Gamma} g_{U}(U)$. The same proof shows that the closed unit ball of $G^{\infty}(U)$ coincides with $\bar{\Gamma} g_{U}(D)$ for each dense subset $D$ of $U$. This shows in particular that $G^{\infty}(U)$ is separable whenever $E$ is separable, a result noticed already by J. M. Ansemil and S. Dineen [1].

2.3. Proposition. Let $E$ be a Banach space.

(a) If $U$ is a bounded open set in $E$, then $E$ is topologically isomorphic to a complemented subspace of $G^{\infty}(U)$.

(b) If $U$ is the open unit ball of $E$, then $E$ is isonetrically isomorphic to a 1-complemented subspace of $G^{\infty}(U)$.

Proof. (a) By Theorem 2.1 there exists $T \in L\left(G^{\infty}(U) ; E\right)$ such that $T$ 。 $g_{U}(x)=x$ for every $x \in U$. Fix $a \in U$ and let $S=P^{1} g_{U}(a) \in L\left(E ; G^{\infty}(U)\right)$. Given $t \in E$, choose $r>0$ such that $a+\zeta t \in U$ for all $\zeta \in \mathbb{C}$ with $|\zeta| \leq r$. By the Cauchy integral formula

$$
S(t)=\frac{1}{2 \pi i} \int_{|\zeta|=r} \frac{g_{U}(a+\zeta t)}{\zeta^{2}} d \zeta,
$$

and therefore

$$
T \circ S(t)=\frac{1}{2 \pi i} \int_{|\zeta|=r} \frac{a+\zeta t}{\zeta^{2}} d \zeta=t .
$$

(b) If $U$ is the open unit ball of $E$, then it follows from Theorem 2.1 that $\|T\|=1$. If we take $a=0$ in (a) then it follows from the Cauchy integral formula that $\|S\| \leq 1$. Hence

$$
\|t\|=\|T \circ S(t)\| \leq\|S(t)\| \leq\|t\|,
$$

for every $t \in E$, and thus $S$ is an isometry.

By following the pattern of the proof of Theorem 2.1, we can also prove the following result, which has been previously obtained by R. Ryan [15] by using tensor product methods.

2.4. Theorem [15]. Let $E$ be a Banach space and let $m \in \mathbb{N}$. Then there are a Banach space $Q\left({ }^{m} E\right)$ and a polynomial $q_{m} \in P\left({ }^{m} E ; Q\left({ }^{m} E\right)\right)$ with the following universal property: For each Banach space $F$ and each polynomial $P \in$ $P\left({ }^{m} E ; F\right)$, there is a unique operator $T_{P} \in L\left(Q\left({ }^{m} E\right) ; F\right)$ such that $T_{P} \circ q_{m}=P$. The correspondence

$$
P \in P\left({ }^{m} E ; F\right) \rightarrow T_{P} \in L\left(Q\left({ }^{m} E\right) ; F\right)
$$


is an isometric isomorphism. These properties characterize $Q\left({ }^{m} E\right)$ uniquely up to an isometric isomorphism.

2.5. Remarks. Our proof of Theorem 2.4 yields also the following facts:

(a) If $B_{m}$ denotes the closed unit ball of $P\left({ }^{m} E\right)$, then $Q\left({ }^{m} E\right)$ is the closed subspace of all $u \in P\left({ }^{m} E\right)^{\prime}$ such that $u \mid\left(B_{m}, \tau_{c}\right)$ is continuous.

(b) $q_{m}: x \in E \rightarrow \delta_{x} \in Q\left({ }^{m} E\right)$ is the evaluation mapping, that is, $\delta_{x}(P)=$ $P(x)$ for every $x \in E$ and $P \in P\left({ }^{m} E\right)$.

(c) The closed unit ball of $Q\left({ }^{m} E\right)$ coincides with the closed, convex, balanced hull $\bar{\Gamma} q_{m}\left(U_{E}\right)$ of $q_{m}\left(U_{E}\right)$. In particular, $q_{m}\left(U_{E}\right)$ generates a dense subspace of $Q\left({ }^{m} E\right)$.

We end this section with the following result, which shows the connection between the spaces $Q\left({ }^{m} E\right)$ and $G^{\infty}(U)$.

\subsection{Proposition. Let $E$ be a Banach space.}

(a) If $U$ is a bounded open set in $E$, then $Q\left({ }^{m} E\right)$ is topologically isomorphic to a complemented subspace of $G^{\infty}(U)$.

(b) If $U$ is the open unit ball of $E$, then $\left.Q{ }^{m} E\right)$ is isometrically isomorphic to a 1-complemented subspace of $G^{\infty}(U)$.

Proof. (a) Fix $a \in U$. By Theorems 2.4 and 2.1 there are operators $S_{m} \in$ $L\left(Q\left({ }^{m} E\right) ; G^{\infty}(U)\right)$ and $T_{m} \in L\left(G^{\infty}(U) ; Q\left({ }^{m} E\right)\right)$ such that the following diagrams are commutative:
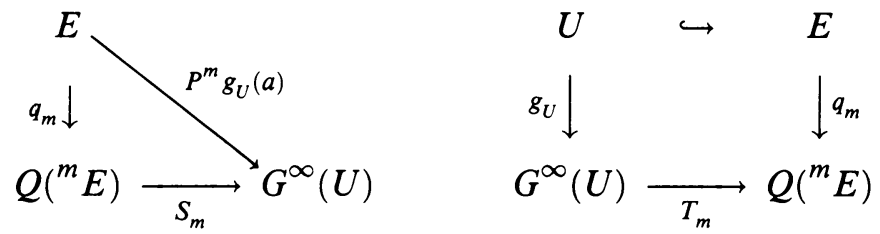

Hence we have that

$$
T_{m} \circ S_{m} \circ q_{m}=T_{m} \circ\left[P^{m} g_{U}(a)\right]=P^{m}\left(T_{m} \circ g_{U}\right)(a)=P^{m} q_{m}(a)=q_{m} .
$$

Since $q_{m}(E)$ generates a dense subspace of $Q\left({ }^{m} E\right)$, it follows that $T_{m} \circ S_{m}(u)=$ $u$ for every $u \in Q\left({ }^{m} E\right)$.

(b) If $U$ is the open unit ball of $E$, then the norm of $q_{m}$ in $H^{\infty}\left(U ; Q\left({ }^{m} E\right)\right)$ equals one, and hence $\left\|T_{m}\right\|=1$. If we take $a=0$ in (a) then it follows from the Cauchy integral formula that the norm of $P^{m} g_{U}(0)$ in $P\left({ }^{m} E ; G^{\infty}(U)\right)$ is not greater than one, and hence $\left\|S_{m}\right\| \leq 1$. Hence

$$
\|u\|=\left\|T_{m} \circ S_{m}(u)\right\| \leq\left\|S_{m}(u)\right\| \leq\|u\|,
$$

for every $u \in Q\left({ }^{m} E\right)$, and thus $S_{m}$ is an isometry.

Observe that $Q\left({ }^{1} E\right)$ is isometrically isomorphic to $E$, and hence Proposition 2.6 includes Proposition 2.3 as a special case.

\section{HOLOMORPHIC MAPPINGS WITH A RELATIVELY COMPACT RANGE}

In this section we translate certain properties of a mapping $f \in H^{\infty}(U ; F)$ into properties of the corresponding operator $T_{f} \in L\left(G^{\infty}(U) ; F\right)$. 
If $X$ is a set and $Y$ is a vector space, then a mapping $f: X \rightarrow Y$ is said to have finite rank if the subspace $N$ of $Y$ generated by $f(X)$ is finite dimensional. In that case we define the rank of $f$ to be the dimension of $N$.

Observe that the subspace of all finite rank mappings $f \in H^{\infty}(U ; F)$ can be canonically identified with $H^{\infty}(U) \otimes F$, and likewise for other spaces of mappings.

Let $P_{f}\left({ }^{m} E ; F\right)$ denote the subspace of all $P \in P\left({ }^{m} E ; F\right)$ of the form $P(x)=\sum_{j=1}^{m}\left[\phi_{j}(x)\right]^{m} b_{j}$, with $\phi_{j} \in E^{\prime}$ and $b_{j} \in F$. Let $P_{f}(E ; F)$ denote the algebraic direct sum of the spaces $P_{f}\left({ }^{m} E ; F\right)$, with $m \in \mathbb{N}_{0}$. The members of $P_{f}(E ; F)$ are called continuous polynomials of finite type. Observe that each $P \in P_{f}(E ; F)$ has finite rank.

As an immediate consequence of Theorems 2.1 and 2.4 we get the following result.

3.1. Proposition. Let $E$ and $F$ be Banach spaces, let $U$ be an open set in $E$, and let $m \in \mathbb{N}$.

(a) A mapping $f \in H^{\infty}(U ; F)$ has finite rank if and only if the corresponding operator $T_{f} \in L\left(G^{\infty}(U) ; F\right)$ has finite rank. In that case $\operatorname{rank} f=\operatorname{rank} T_{f}$.

(b) A polynomial $P \in P\left({ }^{m} E ; F\right)$ has finite rank if and only if the corresponding operator $T_{P} \in L\left(Q\left({ }^{m} E\right) ; F\right)$ has finite rank. In that case $\operatorname{rank} P=\operatorname{rank} T_{P}$.

By a result of K. Floret [7], the operators of rank $\leq n$ in $L\left(G^{\infty}(U) ; F\right)$ form a closed subset of $L\left(G^{\infty}(U) ; F\right)$. Whence it follows that the mappings of rank $\leq n$ in $H^{\infty}(U ; F)$ form a closed subset of $H^{\infty}(U ; F)$. These results are true, not only for the norm topology, but for other suitable topologies as well. A. Chiacchio et al. [3] have used these results to prove that if $F$ is a dual Banach space, then the mappings of rank $\leq n$ in $H^{\infty}(U ; F)$ form a proximinal subset of $l^{\infty}(U ; F)$, the space of all bounded mappings from $U$ into $F$.

Following R. Aron and M. Schottenloher [2], and R. Ryan [15], respectively, we say that a mapping $f \in H(U ; F)$ is compact (resp. weakly compact) if each $x \in U$ has a neighborhood $V_{x} \subset U$ such that $f\left(V_{x}\right)$ is relatively compact (resp. relatively weakly compact) in $F$. After examining the proofs of [2, Proposition 3.4 and 15, Proposition 4.5], we see that if $U$ is connected, then $f \in H(U ; F)$ is compact (resp. weakly compact) if and only if there is a nonvoid open set $V \subset U$ such that $f(V)$ is relatively compact (resp. relatively weakly compact) in $F$. Let $H_{k}(U ; F)$ (resp. $H_{w k}(U ; F)$ ) denote the subspace of all compact (resp. weakly compact) members of $H(U ; F)$. We also write

$$
\begin{aligned}
& H_{k}^{\infty}(U ; F)=H^{\infty}(U ; F) \cap H_{k}(U ; F), \\
& H_{w k}^{\infty}(U ; F)=H^{\infty}(U ; F) \cap H_{w k}(U ; F),
\end{aligned}
$$

and likewise for other spaces of holomorphic mappings.

Finally let $H_{K}^{\infty}(U ; F)$ (resp. $\left.H_{w K}^{\infty}(U ; F)\right)$ denote the subspace of all mappings $f \in H^{\infty}(U ; F)$ which have a relatively compact range (resp. relatively 
weakly compact range). Then clearly we have the inclusions:

$$
\begin{gathered}
H_{K}^{\infty}(U ; F) \subset H_{k}^{\infty}(U ; F) \\
\cap \quad \cap \\
H_{w K}^{\infty}(U ; F) \subset H_{w k}^{\infty}(U ; F),
\end{gathered}
$$

and the following examples show that these inclusions are in general strict.

3.2. Example. Let $\Delta$ denote the open unit disc in $\mathbb{C}$, and let $f \in H^{\infty}\left(\Delta ; c_{0}\right)$ be defined by

$$
f(\zeta)=\left(\zeta^{m}\right)_{m=1}^{\infty}=\sum_{m=1}^{\infty} \zeta^{m} e_{m}
$$

where $e_{m}$ denotes the $m$ th unit vector. Let $0<r<1$. Since $\left|\zeta^{m}\right|<r^{m}$ for every $\zeta \in r \Delta$ and $m \in \mathbb{N}$, we see that the set $f(r \Delta)$ is relatively compact in $c_{0}$ (see [5, p. 15, Exercise 6]), and thus $f \in H_{k}^{\infty}\left(\Delta ; c_{0}\right)$. We claim that $f \notin H_{w K}^{\infty}\left(\Delta ; c_{0}\right)$. Indeed, let $\left(\zeta_{j}\right)$ be a sequence in $\Delta$ which converges to one. If the set $f(\Delta)$ were relatively weakly compact, then the sequence $\left(f\left(\zeta_{j}\right)\right)$ would have a weak cluster point in $c_{0}$. But this is impossible, for a glance at the coordinates of the terms of the sequence $\left(f\left(\zeta_{j}\right)\right)$ shows that the only candidate for a position as cluster point would be the point $(1,1,1, \ldots)$, which does not lie in $c_{0}$. This example shows that in general $H_{K}^{\infty}(U ; F) \neq H_{k}^{\infty}(U ; F)$ and $H_{w K}^{\infty}(U ; F) \neq H_{w k}^{\infty}(U ; F)$.

3.3. Example. Let $E$ be a reflexive, infinite-dimensional Banach space, let $U$ be a bounded, open subset of $E$, and let $f(x)=x$ for every $x \in U$. Then it is clear that $f \in H_{w K}^{\infty}(U ; E)$ and $f \notin H_{k}^{\infty}(U ; E)$. This example shows that in general $H_{K}^{\infty}(U ; F) \neq H_{w K}^{\infty}(U ; F)$ and $H_{k}^{\infty}(U ; F) \neq H_{w k}^{\infty}(U ; F)$.

3.4. Proposition. Let $E$ and $F$ be Banach spaces, let $U$ be an open set in $E$, and let $m \in \mathbb{N}$.

(a) [15] A polynomial $P \in P\left({ }^{m} E ; F\right)$ is compact (resp. weakly compact) if and only if the corresponding operator $T_{P} \in L\left(Q\left({ }^{m} E\right) ; F\right)$ is compact (resp. weakly compact).

(b) A mapping $f \in H^{\infty}(U ; F)$ has a relatively compact range (resp. relatively weakly compact range) if and only if the corresponding operator $T_{f} \in$ $L\left(G^{\infty}(U) ; F\right)$ is compact (resp. weakly compact).

Proof. Since the closed unit ball of $Q\left({ }^{m} E\right)$ coincides with $\bar{\Gamma} q_{m}\left(U_{E}\right)$, (a) follows from the inclusions

$$
P\left(U_{E}\right)=T_{P} \circ q_{m}\left(U_{E}\right) \subset T_{P}\left[\bar{\Gamma} q_{m}\left(U_{E}\right)\right] \subset \bar{\Gamma} T_{P} \circ q_{m}\left(U_{E}\right)=\bar{\Gamma} P\left(U_{E}\right) .
$$

Similarly, since the closed unit ball of $G^{\infty}(U)$ coincides with $\bar{\Gamma} g_{U}(U)$, (b) follows from the inclusions

$$
f(U)=T_{f} \circ g_{U}(U) \subset T_{f}\left[\bar{\Gamma} g_{U}(U)\right] \subset \bar{\Gamma} T_{f} \circ g_{U}(U)=\bar{\Gamma} f(U) .
$$


Proposition 3.4(a) is due to R. Ryan [15], but we have included it here explicitly for the convenience of the reader.

\section{OTHER TOPOLOGIES ON $H^{\infty}(U ; F)$}

Theorem 2.1 tells us that the mapping

$$
f \in H^{\infty}(U ; F) \rightarrow T_{f} \in L\left(G^{\infty}(U) ; F\right)
$$

is an isometric isomorphism. In the next section we shall determine when the space $G^{\infty}(U)$ has the approximation property, and for that purpose it will be useful to have a seminorm description of the unique locally convex topology $\tau_{\gamma}$ on $H^{\infty}(U ; F)$ such that the mapping

$$
f \in\left(H^{\infty}(U ; F) ; \tau_{\gamma}\right) \rightarrow T_{f} \in\left(L\left(G^{\infty}(U) ; F\right), \tau_{c}\right)
$$

is a topological isomorphism.

The corresponding problem for the space $P\left({ }^{m} E ; F\right)$ has already been solved by R. Ryan [15]. His result is essentially the following.

4.1. Theorem [15]. Let $E$ and $F$ be Banach spaces, and let $m \in \mathbb{N}$. Then the mapping

$$
P \in\left(P\left({ }^{m} E ; F\right), \tau_{c}\right) \rightarrow T_{P} \in\left(L\left(Q\left({ }^{m} E\right) ; F\right), \tau_{c}\right)
$$

is a topological isomorphism.

R. Ryan [15] obtained Theorem 4.1 by using tensor product methods. We next give an alternative proof of Theorem 4.1, which will suggest how to tackle the corresponding problem for $H^{\infty}(U ; F)$. The key tool in our proof of Ryan's theorem is the following result, established in [11], and which may be regarded as a generalization of the classical Banach-Dieudonne theorem.

4.2. Theorem [11]. Let $E$ be a Banach space and let $m \in \mathbb{N}$. Then $\tau_{c}$ is the finest topology on $P\left({ }^{m} E\right)$ which coincides with $\tau_{c}$ on each norm bounded subset of $P\left({ }^{m} E\right)$.

With the aid of Theorem 4.2 we can prove the following results which is also due to R. Ryan [15].

4.3. Proposition [15]. Let $E$ be a Banach space, and let $m \in \mathbb{N}$. Then:

(a) $Q\left({ }^{m} E\right)=\left(P\left({ }^{m} E\right), \tau_{c}\right)_{b}^{\prime}$.

(b) The mapping

$$
P \in\left(P\left({ }^{m} E\right), \tau_{c}\right) \rightarrow T_{P} \in Q\left({ }^{m} E\right)_{c}^{\prime}
$$

is a topological isomorphism.

(c) For each compact set $L \subset Q\left({ }^{m} E\right)$, there is a compact set $K \subset E$ such that $L \subset \bar{\Gamma} q_{m}(K)$.

Proof. (a) If follows from Theorem 4.2 that $Q\left({ }^{m} E\right)=\left(P\left({ }^{m} E\right), \tau_{c}\right)^{\prime}$ algebraically. But since $P\left({ }^{m} E\right)$ and $\left(P\left({ }^{m} E\right), \tau_{c}\right)$ have the same bounded sets (see $\left[13\right.$, p. 13, Theorem 2.6]), we conclude that $Q\left({ }^{m} E\right)=\left(P\left({ }^{m} E\right), \tau_{c}\right)_{b}^{\prime}$. 
(b) By Theorem 4.2 a set $N \subset\left(P\left({ }^{m} E\right), \tau_{c}\right)$ is open whenever $N \cap j B_{m}$ is open in $\left(j B_{m}, \tau_{c}\right)$ for every $j \in \mathbb{N}$. Hence an application of [12, Theorem 4.1] shows that the evaluation mapping

$$
\left(P\left({ }^{m} E\right), \tau_{c}\right) \rightarrow\left(\left(P\left({ }^{m} E\right), \tau_{c}\right)_{b}^{\prime}\right)_{c}^{\prime}
$$

is a topological isomorphism. After combining this with (a), the desired conclusion follows.

(c) If $L$ is a compact subset of $Q\left({ }^{m} E\right)$, then the polar $L^{0}$ is a 0 -neighborhood in $Q\left({ }^{m} E\right)_{c}^{\prime}$ and then by (b), the set

$$
\left\{P \in P\left({ }^{m} E\right): T_{P} \in L^{0}\right\}
$$

is a 0 -neighborhood in $\left(P\left({ }^{m} E\right), \tau_{c}\right)$. Hence there is a compact set $K \subset E$ such that

$$
\left\{P \in P\left({ }^{m} E\right): \sup _{K}|P| \leq 1\right\} \subset\left\{P \in P\left({ }^{m} E\right): \sup _{L}\left|T_{p}\right| \leq 1\right\} .
$$

After writing $P=T_{P} \circ q_{m}$ we conclude that $q_{m}(K)^{0} \subset L^{0}$ and therefore

$$
L \subset L^{00} \subset q_{m}(K)^{00}=\bar{\Gamma} q_{m}(K) .
$$

This completes the proof of Proposition 4.3.

Proof of Theorem 4.1. It follows from Proposition 4.3(c) that the mapping in Theorem 4.1 is continuous. And since the inverse mapping is clearly continuous, the proof of Theorem 4.1 is complete.

Motivated by Theorem 4.2 and by the definition of $G^{\infty}(U)$ we introduce the following auxiliary topology. Let $\tau_{b c}$ denote the finest locally convex topology on $H^{\infty}(U)$ which coincides with $\tau_{c}$ on $B_{U}$, or equivalently, on each scalar multiple of $B_{U}$. Clearly $\tau_{b c}$ exists: the convex, balanced sets $N \subset H^{\infty}(U)$ such that $N \cap \lambda B_{U}$ is a 0-neighborhood in $\left(\lambda B_{U}, \tau_{c}\right)$ for every $\lambda>0$, form a 0 -neighborhood base for $\tau_{b c}$. We shall refer to $\tau_{b c}$ as the topology of bounded compact convergence. We shall prove the following theorem.

4.4 Theorem. Let $U$ be an open subset of a Banach space $E$. Then $\tau_{b c}$ is the finest topology on $H^{\infty}(U)$ which coincides with $\tau_{c}$ on each norm bounded subset of $H^{\infty}(U)$.

The proof of Theorem 4.4 will also yield the following theorem.

4.5. Theorem. Let $U$ be an open subset of a Banach space $E$. Then the topology of $\left(H^{\infty}(U), \tau_{b c}\right)$ is generated by all the seminorms of the form

$$
p(f)=\sup _{j} \alpha_{j}\left|f\left(x_{j}\right)\right|,
$$

where $\left(x_{j}\right)$ varies over all sequences in $U$, and $\left(\alpha_{j}\right)$ varies over all sequences of positive numbers tending to zero.

The key to the proof of Theorems 4.4 and 4.5 is the following lemma. 
4.6. Lemma. Let $U$ be an open subset of a Banach space $E$. Let $N$ be a subset of $H^{\infty}(U)$ containing the origin and such that $N \cap j B_{U}$ is open in $\left(j B_{U}, \tau_{c}\right)$ for every $j \in \mathbb{N}$. Then there exist $\delta>0$ and a sequence $\left(A_{j}\right)_{j=0}^{\infty}$ of finite subsets of $U$ such that

$$
N\left(A_{0} ; \delta\right) \cap \bigcap_{j=1}^{\infty} N\left(A_{j} ; j\right) \subset N,
$$

where, for each $A \subset U$ and $r>0, N(A ; r)$ denotes the set

$$
N(A ; r)=\left\{f \in H^{\infty}(U): \sup _{A}|f| \leq r\right\} .
$$

Proof. By hypothesis $N \cap B_{U}$ is open in $B_{U}$ for the compact-open topology, and therefore for the norm topology. Whence there exists $\delta>0$ such that

$$
\delta B_{U} \subset N \text {. }
$$

We claim that there exists a finite set $A_{0} \subset U$ such that

$$
B_{U} \cap N\left(A_{0} ; \delta\right) \subset N \text {. }
$$

Otherwise the sets $\left(B_{U} \backslash N\right) \cap N(A ; \delta)$ would be nonvoid for every finite set $A \subset U$. These sets are closed in $\left(B_{U} \backslash N, \tau_{c}\right)$ and have the finite intersection property. Since the set $B_{U} \backslash N$ is a closed, and therefore compact, subset of $\left(B_{U}, \tau_{c}\right)$, we conclude that the intersection

$$
\bigcap_{A}\left(B_{U} \backslash N\right) \cap N(A ; \delta)
$$

would be nonvoid. But if $f$ belongs to this intersection, we see that $f \in$ $\delta B_{U} \backslash N$, contradicting (4.1), and thus proving (4.2). Proceeding by induction we can show the existence of a sequence $\left(A_{j}\right)_{j=1}^{\infty}$ of finite subsets of $U$ such that

$$
k B_{U} \cap N\left(A_{0} ; \delta\right) \cap \bigcap_{j=1}^{k-1} N\left(A_{j} ; j\right) \subset N,
$$

for every $k=2,3, \ldots$. Since $H^{\infty}(U)=\bigcup_{k=1}^{\infty} k B_{U}$, the desired conclusion follows.

Proof of Theorem 4.4. To begin with we claim that if $\left(K_{j}\right)_{j=1}^{\infty}$ is a sequence of compact subsets of $U$, and $\left(r_{j}\right)_{j=1}^{\infty}$ is a sequence of positive numbers tending to infinity, then the set

$$
\bigcap_{j=1}^{\infty} N\left(K_{j} ; r_{j}\right)
$$

is a 0 -neighborhood in $\left(H^{\infty}(U), \tau_{b c}\right)$. Indeed, given $\lambda>0$, choose $k \in \mathbb{N}$ such that $r_{j}>\lambda$ for every $j>k$. Then

$$
\bigcap_{j=1}^{\infty} N\left(K_{j} ; r_{j}\right) \cap \lambda B_{U}=\bigcap_{j=1}^{k} N\left(K_{j} ; r_{j}\right) \cap \lambda B_{U},
$$

and our claim has been proved. 
Now, let $N$ be a subset of $H^{\infty}(U)$ such that $N \cap \lambda B_{U}$ is open in $\left(\lambda B_{U}, \tau_{c}\right)$ for every $\lambda>0$. To prove the theorem it suffices to show that $N$ is a neighborhood of $f$ in $\left(H^{\infty}(U), \tau_{b c}\right)$ for each $f \in N$. If $f=0$ then the desired conclusion follows at once from Lemma 4.6 and the claim that we have just proved. Finally, the case where $f \in N$ is arbitrary can be reduced to the case $f=0$ by proving that $(N-f) \cap \lambda B_{U}$ is open in $\left(\lambda B_{U}, \tau_{c}\right)$ for every $\lambda>0$. Now, given $\lambda>0$ choose $\mu>\lambda$ such that

$$
\lambda B_{U}+f \subset \mu B_{U} .
$$

By hypothesis there exists an open set $M$ in $\left(H^{\infty}(U), \tau_{c}\right)$ such that

$$
N \cap \mu B_{U}=M \cap \mu B_{U} \text {. }
$$

Hence

$$
\begin{aligned}
(N-f) \cap \lambda B_{U} & =N \cap\left(\lambda B_{U}+f\right)-f \\
& =M \cap\left(\lambda B_{U}+f\right)-f=(M-f) \cap \lambda B_{U},
\end{aligned}
$$

proving that $(N-f) \cap \lambda B_{U}$ is open in $\left(\lambda B_{U}, \tau_{c}\right)$. This completes the proof of the theorem.

Proof of Theorem 4.5. In the course of the proof of Theorem 4.4 we have shown that the sets of the form

$$
N=\bigcap_{j=1}^{\infty} N\left(A_{j} ; r_{j}\right),
$$

where $\left(A_{j}\right)$ varies over all sequences of finite subsets of $U$, and $\left(r_{j}\right)$ varies over all sequences of positive numbers tending to infinity, form a 0 -neighborhood base in $\left(H^{\infty}(U), \tau_{b c}\right)$. Since the Minkowski functional of $N$ is the seminorm

$$
p_{N}(f)=\sup _{j}\left(r_{j}^{-1} \sup _{A_{j}}|f|\right),
$$

the desired conclusion follows at once.

We should remark that Theorem 4.4 follows also from the theory of Saks spaces (see [4, p. 48, Corollary 4.2]), but the proof that we have given here has the advantage of yielding, as a bonus, a proof of Theorem 4.5 . In any case, what lies behind each of the proofs is the idea of the proof of the classical Banach-Dieudonné theorem.

4.7. Proposition. Let $U$ be an open subset of a Banach space E. Then:

(a) $H^{\infty}(U)$ and $\left(H^{\infty}(U), \tau_{b c}\right)$ have the same bounded sets.

(b) $G^{\infty}(U)=\left(H^{\infty}(U), \tau_{b c}\right)_{b}^{\prime}$.

(c) The mapping

$$
f \in\left(H^{\infty}(U), \tau_{b c}\right) \rightarrow T_{f} \in G^{\infty}(U)_{c}^{\prime}
$$

is a topological isomorphism. 
(d) For each compact set $L \subset G^{\infty}(U)$, there are a sequence $\left(x_{j}\right) \subset U$ and a sequence $\left(\alpha_{j}\right) \in c_{0}$ with $\alpha_{j}>0$ such that

$$
L \subset \bar{\Gamma}\left\{\alpha_{j} g_{U}\left(x_{j}\right): j \in \mathbb{N}\right\} .
$$

Proof. (a) It follows from the definitions of $G^{\infty}(U)$ and $\tau_{b c}$ that $G^{\infty}(U)=$ $\left(H^{\infty}(U), \tau_{b c}\right)^{\prime}$ algebraically. Hence a set $\left(f_{\alpha}\right) \subset\left(H^{\infty}(U), \tau_{b c}\right)$ is bounded if and only if it is $\sigma\left(H^{\infty}(U), G^{\infty}(U)\right)$-bounded. But since $H^{\infty}(U)$ is isometrically isomorphic to $G^{\infty}(U)^{\prime}$ and $G^{\infty}(U)$ is a Banach space, this means that $\left(f_{\alpha}\right)$ is norm bounded in $H^{\infty}(U)$.

(b) This follows at once from (a).

(c) By Theorem 4.4 a set $N \subset\left(H^{\infty}(U), \tau_{b c}\right)$ is open whenever $N \cap j B_{U}$ is open in $j B_{U}$, for the induced topology, for every $j \in \mathbb{N}$. Hence an application of [12, Theorem 4.1] shows that the evaluation mapping

$$
\left(H^{\infty}(U), \tau_{b c}\right) \rightarrow\left(\left(H^{\infty}(U), \tau_{b c}\right)_{b}^{\prime}\right)_{c}^{\prime}
$$

is a topological isomorphism. After combining this with (b), we get the desired conclusion. Incidentally, [12, Theorem 4.1] gives another proof of (a).

(d) If $L$ is a compact subset of $G^{\infty}(U)$, then the polar $L^{0}$ is a 0 -neighborhood in $G^{\infty}(U)_{c}^{\prime}$ and then, by (c), the set

$$
\left\{f \in H^{\infty}(U): T_{f} \in L^{0}\right\}
$$

is a 0 -neighborhood in $\left(H^{\infty}(U), \tau_{b c}\right)$. Then by Theorem 4.5 there are a sequence $\left(x_{j}\right) \subset U$ and a sequence $\left(\alpha_{j}\right) \in c_{0}$ with $\alpha_{j}>0$ such that

$$
\left\{f \in H^{\infty}(U): \sup _{j} \alpha_{j}\left|f\left(x_{j}\right)\right| \leq 1\right\} \subset\left\{f \in H^{\infty}(U): \sup _{L}\left|T_{f}\right| \leq 1\right\} .
$$

After writing $f=T_{f} \circ g_{U}$ we conclude that

$$
\left\{\alpha_{j} g_{U}\left(x_{j}\right): j \in \mathbb{N}\right\}^{0} \subset L^{0},
$$

and the desired conclusion follows from the bipolar theorem.

Now we can solve the problem posed at the beginning of this section.

4.8. Theorem. Let $E$ and $F$ be Banach spaces, and let $U$ be an open subset of $E$. Let $\tau_{\gamma}$ denote the locally convex topology on $H^{\infty}(U ; F)$ generated by all the seminorms of the form

$$
p(f)=\sup _{j} \alpha_{j}\left\|f\left(x_{j}\right)\right\|,
$$

where $\left(x_{j}\right)$ varies over all sequences in $U$, and $\left(\alpha_{j}\right)$ varies over all sequences of positive numbers tending to zero. Then the mapping

$$
f \in\left(H^{\infty}(U ; F), \tau_{\gamma}\right) \rightarrow T_{f} \in\left(L\left(G^{\infty}(U) ; F\right), \tau_{c}\right)
$$

is a topological isomorphism

Proof. If $L$ is a compact subset of $G^{\infty}(U)$, then by Proposition 4.7 there are a sequence $\left(x_{j}\right) \subset U$ and a sequence $\left(\alpha_{j}\right) \in c_{0}$ with $\alpha_{j}>0$ such that

$$
L \subset \bar{\Gamma}\left\{\alpha_{j} g_{U}\left(x_{j}\right): j \in \mathbb{N}\right\} .
$$


Hence

$$
\sup _{u \in L}\left\|T_{f} u\right\| \leq \sup _{j}\left\|T_{f}\left(\alpha_{j} g_{U}\left(x_{j}\right)\right)\right\|=\sup _{j} \alpha_{j}\left\|f\left(x_{j}\right)\right\|,
$$

for every $f \in H^{\infty}(U ; F)$, and the mapping $f \rightarrow T_{f}$ is $\tau_{\gamma}-\tau_{c}$-continuous. Since the sequence $\left(\alpha_{j} g_{U}\left(x_{j}\right)\right)$ tends to zero in $G^{\infty}(U)$, the inverse mapping is continuous as well. This completes the proof.

The topologies $\tau_{\gamma}, \tau_{c}$ and $\tau_{b c}$ are related as follows.

4.9. Proposition. Let $E$ and $F$ be Banach spaces, and let $U$ be an open set in $E$. Then:

(a) $\tau_{\gamma} \geq \tau_{c}$ on $H^{\infty}(U ; F)$.

(b) $\tau_{\gamma}$ coincides with $\tau_{c}$ on each norm bounded subset of $H^{\infty}(U ; F)$.

(c) $\tau_{\gamma}=\tau_{b c}$ on $H^{\infty}(U)$.

(d) If $U$ is bounded, then $\tau_{\gamma}$ coincides with $\tau_{c}$ on $P\left({ }^{m} E ; F\right)$, for each $m \in \mathbb{N}$.

Proof. (a) If $K$ is a compact subset of $U$, then by Proposition 4.7 there are a sequence $\left(x_{j}\right) \subset U$ and a sequence $\left(\alpha_{j}\right) \in c_{0}$ with $\alpha_{j}>0$ such that

Hence it follows that

$$
g_{U}(K) \subset \bar{\Gamma}\left\{\alpha_{j} g_{U}\left(x_{j}\right): j \in \mathbb{N}\right\} .
$$

$$
\sup _{x \in K}\|f(x)\| \leq \sup _{j} \alpha_{j}\left\|f\left(x_{j}\right)\right\|
$$

for every $f \in H^{\infty}(U ; F)$.

(b) Let $p(f)=\sup _{j} \alpha_{j}\left\|f\left(x_{j}\right)\right\|$, where $\left(x_{j}\right) \subset U$ and $\left(\alpha_{j}\right) \in c_{0}$, with $\alpha_{j}>$ 0 . Given $\lambda>0$ and $\varepsilon>0$ choose $k \in \mathbb{N}$ such that $2 \alpha_{j} \lambda<\varepsilon$ for every $j>k$. Then for $f, g \in H^{\infty}(U ; F)$ with $\|f\|<\lambda$ and $\|g\|<\lambda$ we have that $p(f-g)<\varepsilon$ whenever $\alpha_{j}\left\|f\left(x_{j}\right)-g\left(x_{j}\right)\right\|<\varepsilon$ for $j=1, \ldots, k$. This shows that the restriction of $p$ to each norm bounded subset of $H^{\infty}(U ; F)$ is $\tau_{c}$-continuous.

(c) This follows from Theorem 4.5 and the definition of $\tau_{\gamma}$.

(d) Let $p(f)=\sup _{j} \alpha_{j}\left\|f\left(x_{j}\right)\right\|$, where $\left(x_{j}\right) \subset U$ and $\left(\alpha_{j}\right) \in c_{0}$, with $\alpha_{j}>0$. Let $\beta_{j}=\sqrt[m]{\alpha_{j}}$ for every $j \in \mathbb{N}$. Then $p(P)=\sup _{j}\left\|P\left(\beta_{j} x_{j}\right)\right\|$ for every $P \in P\left({ }^{m} E ; F\right)$, and thus $p \mid P\left({ }^{m} E ; F\right)$ is $\tau_{c}$-continuous.

From Theorem 4.8 and Proposition 4.9 we get at once the following corollary. 4.10. Corollary. Let $E$ and $F$ be Banach spaces, and let $U$ be an open set in E. Then:

(a) The mapping

$$
T \in\left(L\left(G^{\infty}(U) ; F\right), \tau_{c}\right) \rightarrow T \circ g_{U} \in\left(H^{\infty}(U ; F), \tau_{c}\right)
$$

is continuous.

(b) The restriction of the inverse mapping

$$
f \in\left(H^{\infty}(U ; F), \tau_{c}\right) \rightarrow T_{f} \in\left(L\left(G^{\infty}(U) ; F\right), \tau_{c}\right)
$$

to each norm bounded subset of $H^{\infty}(U ; F)$ is continuous. 
We should remark that assertion (a) in Corollary 4.10 is indeed obvious, whereas assertion (b) is not obvious, but can be proved directly after Theorem 2.1, without recourse to the topologies $\tau_{b c}$ or $\tau_{\gamma}$.

We end this section with an explicit description of the dual of the space $\left(H^{\infty}(U ; F), \tau_{\gamma}\right)$.

4.11. Theorem. Let $E$ and $F$ be Banach spaces, and let $U$ be an open set in $E$. Then the dual of $\left(H^{\infty}(U ; F), \tau_{\gamma}\right)$ consists of all linear functionals of the form

$$
u(f)=\sum_{j=1}^{\infty} \psi_{j} \circ f\left(x_{j}\right),
$$

where $\left(x_{j}\right)$ varies over all sequences in $U$, and $\left(\psi_{j}\right)$ varies over all sequences in $F^{\prime}$ with $\sum_{j=1}^{\infty}\left\|\psi_{j}\right\|<\infty$.

Proof. We first assume that $u$ has such a representation. Since $\sum_{j=1}^{\infty}\left\|\psi_{j}\right\|<$ $\infty$, we can find a sequence $\left(\lambda_{j}\right)$ of positive numbers tending to infinity such that $\sum_{j=1}^{\infty} \lambda_{j}\left\|\psi_{j}\right\|=c<\infty$. Then

$$
|u(f)| \leq \sum_{j=1}^{\infty}\left\|\psi_{j}\right\|\left\|f\left(x_{j}\right)\right\| \leq c \sup _{j} \lambda_{j}^{-1}\left\|f\left(x_{j}\right)\right\|,
$$

for every $f \in H^{\infty}(U ; F)$, proving that $u$ is $\tau_{\gamma}$-continuous.

Conversely, assume that $u$ is a continuous linear functional on the space $\left(H^{\infty}(U ; F), \tau_{\gamma}\right)$. Then there are a sequence $\left(x_{j}\right) \subset U$ and a sequence $\left(\alpha_{j}\right) \in c_{0}$ with $\alpha_{j}>0$ such that

$$
|u(f)| \leq \sup _{j} \alpha_{j}\left\|f\left(x_{j}\right)\right\|
$$

for every $f \in H^{\infty}(U ; F)$. Now, it follows from the very definition of $\tau_{\gamma}$ that the linear mapping

$$
v: f \in\left(H^{\infty}(U ; F), \tau_{\gamma}\right) \rightarrow\left(\alpha_{j} f\left(x_{j}\right)\right)_{j=1}^{\infty} \in c_{0}(F)
$$

is continuous. Since $|u(f)| \leq\|v(f)\|$ for every $f \in H^{\infty}(U ; F)$, we may define a continuous linear functional $w$ on $v\left(H^{\infty}(U ; F)\right)$ by $w(v(f))=u(f)$ for every $f \in H^{\infty}(U ; F)$. By the Hahn-Banach theorem $w$ admits a continuous linear extension to $c_{0}(F)$. Since $c_{0}(F)^{\prime}=l^{1}\left(F^{\prime}\right)$, there is a sequence $\left(\psi_{j}\right) \in$ $l^{1}\left(F^{\prime}\right)$ such that

$$
u(f)=w(v(f))=\sum_{j=1}^{\infty} \alpha_{j} \psi_{j} \circ f\left(x_{j}\right),
$$

for every $f \in H^{\infty}(U ; F)$. This completes the proof.

4.12. Corollary. Let $U$ be an open subset of a Banach space $E$. Then $G^{\infty}(U)$ consists of all linear functionals $u \in H^{\infty}(U)^{\prime}$ of the form

$$
u(f)=\sum_{j=1}^{\infty} \alpha_{j} f\left(x_{j}\right)
$$


where $\left(x_{j}\right)$ varies over all sequences in $U$, and $\left(\alpha_{j}\right)$ varies over all absolutely summable sequences of complex numbers.

Proof. By Propositions 4.7 and 4.9, $G^{\infty}(U)=\left(H^{\infty}(U), \tau_{\gamma}\right)^{\prime}$. Thus it suffices to apply Theorem 4.11 .

\section{THE APPROXIMATION PROPERTY}

In this section we give necessary and sufficient conditions for the spaces $G^{\infty}(U)$ and $H^{\infty}(U)$ to have the approximation property. These are holomorphic analogues of classical results of A. Grothendieck [8, pp. 164-167, Propositions 35 and 36] (see also [10, pp. 32-33, Theorems 1.e.4 and 1.e.5]), which will be used without further reference. Our results in this section complement results of R. Aron and M. Schottenloher [2].

We begin with some auxiliary results.

5.1. Lemma. Let $F$ be a Banach space and let $f(\zeta)=\sum_{m=0}^{\infty} \zeta^{m} c_{m}$ be an $F$ valued holomorphic function on an open disc $D$ centered at the origin. For each $m \in \mathbb{N}_{0}$ let

$$
S_{m} f(\zeta)=\sum_{k=0}^{m} \zeta^{k} c_{k}
$$

and

$$
\sigma_{m} f(\zeta)=\frac{1}{m+1} \sum_{k=0}^{m} S_{k} f(\zeta)=\sum_{k=0}^{m} \frac{m+1-k}{m+1} \zeta^{k} c_{k} .
$$

Then for each $\zeta \in D$ we have that

$$
S_{m} f(\zeta)=\frac{1}{\pi} \int_{-\pi}^{\pi} f\left(e^{i t} \zeta\right) D_{m}(t) d t
$$

and

$$
\sigma_{m} f(\zeta)=\frac{1}{\pi} \int_{-\pi}^{\pi} f\left(e^{i t} \zeta\right) K_{m}(t) d t
$$

where $D_{m}(t)$ and $K_{m}(t)$ denote the classical Dirichlet kernel and Fejer kernel, respectively.

Proof. Since $f\left(r e^{i t}\right)=\sum_{m=0}^{\infty} r^{m} e^{i m t} c_{m}$, we see that

$$
\frac{1}{2 \pi} \int_{-\pi}^{\pi} f\left(r e^{i t}\right) e^{-i k t} d t= \begin{cases}r^{k} c_{k} & \text { for } k=0,1,2, \ldots \\ 0 & \text { for } k=-1,-2, \ldots\end{cases}
$$


Hence for $k=1,2, \ldots$ we get that

$$
\begin{aligned}
r^{k} e^{i k \theta} c_{k} & =\frac{e^{i k \theta}}{2 \pi} \int_{-\pi}^{\pi} f\left(r e^{i t}\right) e^{-i k t} d t+\frac{e^{-i k \theta}}{2 \pi} \int_{-\pi}^{\pi} f\left(r e^{i t}\right) e^{i k t} d t \\
& =\frac{1}{2 \pi} \int_{-\pi}^{\pi} f\left(r e^{i t}\right)\left(e^{i k(\theta-t)}+e^{i k(t-\theta)}\right) d t \\
& =\frac{1}{\pi} \int_{-\pi}^{\pi} f\left(r e^{i t}\right) \cos k(t-\theta) d t \\
& =\frac{1}{\pi} \int_{-\pi}^{\pi} f\left(r e^{i(t+\theta)}\right) \cos k t d t .
\end{aligned}
$$

Thus

$$
S_{m} f\left(r e^{i \theta}\right)=\frac{1}{\pi} \int_{-\pi}^{\pi} f\left(r e^{i(t+\theta)}\right)\left(\frac{1}{2}+\sum_{k=1}^{m} \cos k t\right) d t .
$$

After recalling that

$$
D_{m}(t)=\frac{1}{2}+\sum_{k=1}^{m} \cos k t \quad \text { and } \quad K_{m}(t)=\frac{1}{m+1} \sum_{k=0}^{m} D_{k}(t),
$$

the desired conclusion follows.

5.2. Proposition. Let $E$ and $F$ be Banach spaces, and let $U$ be a balanced open set in $E$. Let $f \in H(U ; F)$ and let

$$
S_{m} f(x)=\sum_{k=0}^{m} P^{k} f(0)(x)
$$

and

$$
\sigma_{m} f(x)=\frac{1}{m+1} \sum_{k=0}^{m} S_{k} f(x)=\sum_{k=0}^{m} \frac{m+1-k}{m+1} P^{k} f(0)(x) .
$$

Then:

(a) $S_{m} f \rightarrow f$ and $\sigma_{m} f \rightarrow f$ in $\left(H(U ; F), \tau_{c}\right)$.

(b) For each $x \in U$ we have that

$$
S_{m} f(x)=\frac{1}{\pi} \int_{-\pi}^{\pi} f\left(e^{i t} x\right) D_{m}(t) d t
$$

and

$$
\sigma_{m} f(x)=\frac{1}{\pi} \int_{-\pi}^{\pi} f\left(e^{i t} x\right) K_{m}(t) d t .
$$

(c) If $\|f(x)\| \leq c$ for every $x \in U$, then $\left\|\sigma_{m} f(x)\right\| \leq c$ for every $x \in U$ and $m \in \mathbb{N}$, and $\sigma_{m} f \rightarrow f$ in $\left(H^{\infty}(U ; F), \tau_{\gamma}\right)$.

Proof. (a) is clear. To show (b) fix $x \in U$ and apply the preceding lemma to the function

$$
g(\zeta)=f(\zeta x)=\sum_{m=0}^{\infty} \zeta^{m} P^{m} f(0)(x)
$$


The first assertion in (c) follows from (b) and the facts that $K_{m}(t) \geq 0$ for every $t$ and $\frac{1}{\pi} \int_{-\pi}^{\pi} K_{m}(t) d t=1$ (see [17, p. 45]). Finally, the second assertion in (c) follows from (a) and Proposition 4.9.

The following lemma is well known, and is easy to prove anyway.

5.3. Lemma. If $E$ is a Banach space with the approximation property, then $P_{f}\left({ }^{m} E ; F\right)$ is $\tau_{c}$-dense in $P\left({ }^{m} E ; F\right)$ for each Banach space $F$ and each $m \in \mathbb{N}$.

5.4. Theorem. Let $E$ be a Banach space and let $U$ be a balanced, bounded, open set in $E$. Then the following conditions are equivalent:

(a) $E$ has the approximation property.

(b) For each Banach space $F, P_{f}(E ; F)$ is $\tau_{\gamma}$-dense in $H^{\infty}(U ; F)$.

(c) For each Banach space $F, H^{\infty}(U) \otimes F$ is $\tau_{\gamma}$-dense in $H^{\infty}(U ; F)$.

(d) $g_{U}$ lies in the $\tau_{\gamma}$-closure of $H^{\infty}(U) \otimes G^{\infty}(U)$.

(e) $G^{\infty}(U)$ has the approximation property.

(f) For each Banach space $F$ and each open set $V \subset F, H^{\infty}(V) \otimes E$ is $\tau_{\gamma}$-dense in $H^{\infty}(V ; E)$.

(g) For each Banach space $F$ and each open set $V \subset F, H^{\infty}(V) \otimes E$ is norm-dense in $H_{K}^{\infty}(V ; E)$.

(h) The identity mapping on $U$ lies in the $\tau_{\gamma}$-closure of $H^{\infty}(U) \otimes E$.

Proof. (a) $\Rightarrow\left(\right.$ b) : Let $f \in H^{\infty}(U ; F)$ and let $p$ be a continuous seminorm on $\left(H^{\infty}(U ; F), \tau_{\gamma}\right)$. By Proposition 5.2 we can find $P \in P(E ; F)$ such that $p(P-f)<\frac{1}{2}$. Since, by Proposition 4.9, $\tau_{\gamma}$ coincides with $\tau_{c}$ on $P\left({ }^{m} E ; F\right)$ for each $m \in \mathbb{N}$, repeated applications of Lemma 5.3 yield a polynomial $Q \in$ $P_{f}(E ; F)$ such that $p(Q-P)<\frac{1}{2}$. Thus $p(Q-f)<1$ and $(\mathrm{b})$ has been proved.

(b) $\Rightarrow$ (c) and (c) $\Rightarrow$ (d): Obvious.

(d) $\Rightarrow$ (e): It follows from (d), Proposition 3.1 and Theorem 4.8 that the identity operator on $G^{\infty}(U)$ lies in the $\tau_{c}$-closure of $G^{\infty}(U)^{\prime} \otimes G^{\infty}(U)$. Hence $G^{\infty}(U)$ has the approximation property.

(e) $\Rightarrow($ a): This follows from Proposition 2.3.

(a) $\Rightarrow(\mathrm{f})$ : Since $E$ has the approximation property, $G^{\infty}(V)^{\prime} \otimes E$ is $\tau_{c^{-}}$ dense in $L\left(G^{\infty}(V) ; E\right)$. By applying Proposition 3.1 and Theorem 4.8 we get (f).

(f) $\Rightarrow$ (a): It follows from (f), Proposition 3.1 and Theorem 4.8 that $G^{\infty}\left(U_{F}\right)^{\prime} \otimes E$ is $\tau_{c}$-dense in $L\left(G^{\infty}\left(U_{F}\right) ; E\right)$ for each Banach space $F$. To show that $E$ has the approximation property it suffices to prove that $F^{\prime} \otimes E$ is $\tau_{c}$-dense in $L(F ; E)$ for each Banach space $F$. Let $A \in L(F ; E)$ be given. By Proposition 2.3 there are operators $S \in L\left(F ; G^{\infty}\left(U_{F}\right)\right)$ and $T \in$ $L\left(G^{\infty}\left(U_{F}\right) ; F\right)$ such that $T \circ S(y)=y$ for every $y \in F$. Then $A \circ T \in$ $L\left(G^{\infty}\left(U_{F}\right) ; E\right)$ and therefore there is a net $\left(B_{\alpha}\right) \subset G^{\infty}\left(U_{F}\right)^{\prime} \otimes E$ which converges to $A \circ T$ for $\tau_{c}$. Whence it follows that the net $\left(B_{\alpha} \circ S\right)$ lies in $F^{\prime} \otimes E$ and converges to $A \circ T \circ S=A$ for $\tau_{c}$. 
(a) $\Rightarrow(\mathrm{g})$ : Since $E$ has the approximation property, $G^{\infty}(V)^{\prime} \otimes E$ is normdense in $L_{k}\left(G^{\infty}(V) ; E\right)$. By applying Propositions 3.1 and 3.4, and Theorem 2.1 , we get $(\mathrm{g})$.

(g) $\Rightarrow$ (a): It follows from (g), Propositions 3.1 and 3.4, and Theorem 2.1, that $G^{\infty}\left(U_{F}\right)^{\prime} \otimes E$ is norm-dense in $L_{k}\left(G^{\infty}\left(U_{F}\right) ; E\right)$ for each Banach space $f$. Then, by imitating the proof that $(\mathrm{f}) \Rightarrow(\mathrm{a})$, we can show that $F^{\prime} \otimes E$ is norm-dense in $L_{k}(F ; E)$ for each Banach space $F$. This shows (a).

(c) $\Rightarrow(\mathrm{h})$ : Obvious.

(h) $\Rightarrow$ (c): Let $f \in H^{\infty}(U ; F)$ and let $p$ be a continuous seminorm on $\left(H^{\infty}(U ; F), \tau_{\gamma}\right)$. We wish to find $g \in H^{\infty}(U) \otimes F$ such that $p(g-f)<1$. We may assume that

$$
p(h)=\sup _{j} \alpha_{j}\left\|h\left(x_{j}\right)\right\|,
$$

for every $h \in H^{\infty}(U ; F)$, where $\left(x_{j}\right) \subset U$ and $\left(\alpha_{j}\right) \in c_{0}$ with $\alpha_{j}>0$. By Proposition 5.2 there exists $P \in P(E ; F)$ such that

$$
p(P-f)<\frac{1}{2} \text {. }
$$

Write $P=P_{0}+\cdots+P_{m}$, with $P_{k} \in P\left({ }^{k} E ; F\right)$. Certainly $P_{0} \in H^{\infty}(U) \otimes F$. For each $k=1, \ldots, m$ we shall find $u_{k} \in H^{\infty}(U) \otimes E$ such that

$$
p\left(P_{k} \circ u_{k}-P_{k}\right)<\frac{1}{2 m} \text {. }
$$

It will then follow that

$$
P_{0}+\sum_{k=1}^{m} P_{k} \circ u_{k} \in H^{\infty}(U) \otimes F
$$

and

$$
p\left(P_{0}+\sum_{k=1}^{m} P_{k} \circ u_{k}-f\right) \leq \sum_{k=1}^{m} p\left(P_{k} \circ u_{k}-P_{k}\right)+p(P-f)<1,
$$

thus proving (c). Now, fix $k$ with $1 \leq k \leq m$, let $\beta_{j}=\sqrt[k]{\alpha_{j}}$ for every $j \in \mathbb{N}$ and let $K=\left\{\beta_{j} x_{j}: j \in \mathbb{N}\right\} \cup\{0\}$. Since $K$ is compact, we can find $\delta>0$ such that

$$
\left\|P_{k}(y)-P_{k}(x)\right\|<\frac{1}{2 m},
$$

whenever $x \in K$ and $\|y-x\|<\delta$. By (h) we can find $u_{k} \in H^{\infty}(U) \otimes E$ such that

$$
\sup _{j} \beta_{j}\left\|u_{k}\left(x_{j}\right)-x_{j}\right\|<\delta \text {. }
$$

Hence

$$
\begin{aligned}
p\left(P_{k} \circ u_{k}-P_{k}\right) & =\sup _{j} \alpha_{j}\left\|P_{k} \circ u_{k}\left(x_{j}\right)-P_{k}\left(x_{j}\right)\right\| \\
& =\sup _{j}\left\|P_{k}\left(\beta_{j} u_{k}\left(x_{j}\right)\right)-P_{k}\left(\beta_{j} x_{j}\right)\right\|<\frac{1}{2 m}
\end{aligned}
$$


and $u_{k}$ satisfies (5.2). Thus $(\mathrm{h}) \Rightarrow(\mathrm{c})$ and the proof of the theorem is complete. 5.5. Corollary. $A$ Banach space $E$ has the approximation property if and only if $Q\left({ }^{m} E\right)$ has the approximation property for each $m \in \mathbb{N}$.

5.6. Proposition. Let $U$ be an open subset of a Banach space E. Then $H^{\infty}(U)$ has the approximation property if and only if, for each Banach space $F, H^{\infty}(U) \otimes$ $F$ is norm-dense in $H_{K}^{\infty}(U ; F)$.

Proof. $G^{\infty}(U)^{\prime}$ has the approximation property if and only if, for each Banach space $F, G^{\infty}(U)^{\prime} \otimes F$ is norm-dense in $L_{k}\left(G^{\infty}(U) ; F\right)$. Thus the desired conclusion follows from Propositions 3.1 and 3.4, and Theorem 2.1.

Since it is still unknown whether $H^{\infty}(\Delta)$ has the approximation property, Proposition 5.6 may be of some use in this connection.

We next state two results on the metric approximation property, which are parallel to Theorem 5.4 and Corollary 5.5.

5.7. Proposition. Let $E$ be a Banach space, and let $U$ be the open unit ball of $E$. Then the following conditions are equivalent:

(a) E has the metric approximation property.

(b) For each Banach space $F$, the polynomials in $P_{f}(E ; F)$ which are bounded by one on $U$ in norm, are $\tau_{c}$-dense in the closed unit ball of $H^{\infty}(U ; F)$.

(c) For each Banach space $F$, the mappings of norm $\leq 1$ in $H^{\infty}(U) \otimes F$ are $\tau_{c}$-dense in the closed unit ball of $H^{\infty}(U ; F)$.

(d) $g_{U}$ lies in the $\tau_{c}$-closure of the mappings of norm $\leq 1$ in $H^{\infty}(U) \otimes$ $G^{\infty}(U)$.

(e) $G^{\infty}(U)$ has the metric approximation property.

(f) For each Banach space $F$ and each open set $V \subset F$, the mappings of norm $\leq 1$ in $H^{\infty}(V) \otimes E$ are $\tau_{c}$-dense in the closed unit ball of $H^{\infty}(V ; E)$.

(g) The identity mapping on $U$ lies in the $\tau_{c}$-closure of the mappings of norm $\leq 1$ in $H^{\infty}(U) \otimes E$.

5.8. Corollary. $A$ Banach space $E$ has the metric approximation property if and only if $Q\left({ }^{m} E\right)$ has the metric approximation property for each $m \in \mathbb{N}$.

We should remark that the proof of Proposition 5.7 requires much less machinery than the proof of Theorem 5.4. Indeed, the proof of Theorem 5.4 relies heavily on Theorem 4.8, which was the ultimate goal of $\S 4$, whereas the proof of Proposition 5.7 relies only on Corollary 4.10 , which, as we have remarked already, can be proved directly after Theorem 2.1 , without recourse to the topologies $\tau_{b c}$ or $\tau_{\gamma}$.

One can show that if a Banach space $E$ has the $\lambda$-bounded approximation property for some $\lambda \geq 1$, then $Q\left({ }^{m} E\right)$ has the $\lambda^{m}$-bounded approximation property for each $m \in \mathbb{N}$. However, I have been unable to solve the following problem.

5.9. Problem. If $E$ is a Banach space with the bounded approximation property, does $G^{\infty}\left(U_{E}\right)$ have the bounded approximation property? 
If $E$ is a Banach space with the bounded approximation property, J. Lindenstrauss and L. Tzafriri [10, p. 42, Problem 1.e.21] have asked whether there exists an equivalent norm \|\|$\cdot \|$. on $E$ such that $(E,\||\cdot|\|)$ has the metric approximation property. In view of Proposition 5.7, this problem is equivalent to the following.

5.10. Problem. If $E$ is a Banach space with the bounded approximation property, does there exist a convex, balanced, bounded, open set $U \subset E$ such that $G^{\infty}(U)$ has the metric approximation property?

Added in proof. A. Pelczynski has observed that Proposition 2.3 is closely connected with a result of $\mathrm{H}$. Milne [Banach space properties of uniform algebras, Bull. London Math. Soc. 4 (1972), 323-326], namely that every complex Banach space $E$ is isometrically isomorphic to a complemented subspace of a suitable uniform algebra. Indeed, by applying Proposition 2.3 to the dual of $E$, taking dual mappings, and restricting to $E$, one immediately recovers Milne's result.

\section{REFERENCES}

1. J. M. Ansemil and S. Dineen, Locally determining sequences in infinite dimensional spaces, Note Mat. 7 (1987), 41-45.

2. A. Aron and M. Schottenloher, Compact holomorphic mappings on Banach spaces and the approximation property, J. Funct. Anal. 21 (1976), 7-30.

3. A. Chiacchio, M. Matos, and S. Roversi, On best approximation by rational and holomorphic mappings between Banach spaces, J. Approx. Theory 58 (1989), 334-351.

4. J. B. Cooper, Saks spaces and applications to functional analysis, 2nd ed., North-Holland Math. Studies, vol. 139, North-Holland, Amsterdam, 1987.

5. J. Diestel, Sequences and series in Banach spaces, Graduate Texts in Math., vol. 92, Springer, Berlin, 1984.

6. S. Dineen, Complex analysis in locally convex spaces, North.-Holland Math. Studies, vol. 57, North-Holland, Amsterdam, 1981.

7. K. Floret, Elementos de posto finito em produtos tensoriais topológicos, Atas $24^{\circ}$ Seminário Brasileiro de Análise, Sociedade Brasileira de Matematica, 1986, pp. 189-195.

8. A. Grothendieck, Produits tensoriels topologiques et espaces nucléaires, Mem. Amer. Math. Soc. No. 16 (1955).

9. J. Horváth, Topological vector spaces and distributions, Vol. I, Addison-Wesley, Reading, Mass., 1966.

10. J. Lindenstrauss and L. Tzafriri, Classical Banach spaces. I, Ergeb. Math. Grenzgeb., Bd. 92, Springer, Berlin, 1977.

11. J. Mujica, Complex homomorphisms of the algebras of holomorphic functions on Fréchet spaces, Math. Ann. 241 (1979), 73-82.

12. _ A Banach-Dieudonné theorem for germs of holomorphic functions, J. Funct. Anal. $\mathbf{5 7}$ (1984), 31-84.

13. __ Complex analysis in Banach spaces, North-Holland Math. Studies, vol. 120, NorthHolland, Amsterdam, 1986.

14. K. F. Ng, On a theorem of Dixmier, Math. Scand. 29 (1971), 279-280.

15. R. Ryan, Applications of topological tensor products to infinite dimensional holomorphy, Ph.D. Thesis, Trinity College, Dublin, 1980. 
16. H. H. Schaefer, Topological vector spaces, 3rd printing, Graduate Texts in Math., vol. 3, Springer, Berlin, 1971.

17. A. Zygmund, Trigonometrical series, Dover, New York, 1955.

Instituto de Matemática, Universidade Estadual de Campinas, Caixa Postal 6065, 13.081-CAMPINAS, SP, BRAZIL 